EPJ Web of Conferences 92,02113 (2015)

DOI: $10.1051 /$ epjconf/ 20159202113

(C) Owned by the authors, published by EDP Sciences, 2015

\title{
Experimental and Numerical Investigation of Flow Structures around Cylindrical Bluff Bodies
}

\author{
Sercan Yagmur ${ }^{1, a}$, Sercan Dogan ${ }^{1}$, Muharrem H. Aksoy ${ }^{1}$, Eyub Canli ${ }^{1}$, Muammer Ozgoren ${ }^{1}$ \\ ${ }^{I}$ Selcuk University, Engineering Faculty, Mechanical Engineering Department, 42250 Konya, Turkey
}

\begin{abstract}
The understanding and quantitative prediction of velocity and pressure fluctuations in turbulent flows around such bluff bodies have been evolving over the years. The main aim of the present work is to investigate experimentally and numerically the flow field in the wake region of different bluff bodies such as circular, square and triangle cross section cylinders placed horizontally perpendicular to the uniform flow. The experimental studies were performed by Particle Image Velocimetry (PIV) method in an open water channel at Reynolds numbers 5000 and 10000 defined according to the characteristic lengths of the cylinders in the facilities of Selcuk University of Advanced Technology Research and Application Center in Turkey. The experimental results are compared to the numerical results obtained by means of transient simulation with LES turbulence model of ANSYS-Fluent Software. It is shown that the numerical and experimental results have a good agreement in respect of the instantaneous and time-averaged flow field patterns of vorticity, velocity component streamwise direction and streamline topology. In addition, drag coefficient of the geometries were also numerically calculated. For all geometries the wake length in $\mathrm{x}$ and $\mathrm{y}$ directions and size of the foci of the streamlines are decreasing by increasing Reynolds numbers in time-averaged results. The time-averaged flow patterns of both experimental and numerical results have considerable symmetry with respect to the centerline of each cylinder. Contours of the time-averaged stream wise velocity for $\mathrm{Re}=10000$ demonstrate that the stagnation point around the symmetry plane moves further upstream for all cylinders in accordance with $\mathrm{Re}=5000$. The maximum drag coefficient value was yielded for the square cross-section cylinder as 1.78 due to the sharp-edged geometry.
\end{abstract}

\section{Introduction}

The motion of fluid passing through different crosssection cylinders is encountered in several industrial applications, as well as offshore and environmental settings, including tall buildings and structures such as bridges, chimneys, trash racks, cooling towers, heat exchangers, vortex flow meters etc. When fluid flows around any bluff body, the vortices are alternatively shed and a well-known Karman vortex street is formed in the wake of the cylinder, which can cause structural damage as a result of regular and irregular surface loading [1].

The understanding and quantitative prediction of velocity and pressure fluctuations in turbulent flows around such bluff bodies have been evolving over the years. Some of the examples of the numerous experimental studies in this field are completed by Kim and Geropp (1998), Lam et al (2003), Perrin et al (2005), Ozgoren (2006), Ozgoren et al. (2011), Ozgoren and Dogan (2011), Ozgoren (2013) and Uffinger et al. (2013). For example, Ozgoren focused on the generation of vertical structures of the near-wake region appearing from flow passing the different cross sectional cylinders for $550 \leq \mathrm{Re} \leq 3400$ in order to explain the physical mechanisms of the flow structure. He concluded that the values of Strouhal number, as well as the wake patterns, are functions of the cross-section of the cylinders and Reynolds numbers [1]. Okajima carried out an experimental study of flow past the SC as well as the rectangular cylinder for $70 \leq \operatorname{Re} \leq 20000$ to determine the vortex shedding frequencies. The results showed that there was an abrupt change in Strouhal number when the aspect ratio of the cylinder was reduced to the range 2-3. Strouhal numbers for the $\mathrm{SC}$ with an increasing angle of incidence from $0 \circ$ to $45^{\circ}$ were examined using a hot-wire probe in a closed circuit wind-tunnel for $\mathrm{Re}=10000$ [2]. Kim and Geropp experimentally studied the ground effect on the flow around the bluff bodies with moving-belt technique. The pressure distribution on the body as well as the lift and drag coefficients are affected significantly by the interaction of the moving ground and fluid. The comparison of the experimental results for a moving and a stationary ground plane show strong local deviations, especially on the bottom side of the body and on the wake region [3].

Lam et al. obtained the wake patterns and flow fields of four cylinders arranged in a square configuration under steady laminar uniform cross-flow using a laser-induced fluorescent (LIF) visualization technique and a particle image velocimetry (PIV) technique. Two major flow

\footnotetext{
a Sercan YAGMUR: syagmur@selcuk.edu.tr
} 
patterns which give rise to strong fluctuating forces and serious vibrations are observed. One major flow pattern is the impingement by an oncoming vortex directly and the other is the formation of a jet flow between the relatively stagnated wake of the upstream and downstream cylinder [4]. Ozgoren represents the results of an experimental study on the flow structure around a single sphere and three spheres in an equilateral-triangular arrangement. It was observed that a continuous flow development involving shearing phenomena and the interactions of shedding vortices caused a high rate of fluctuations over the whole flow field although most of the time-averaged flow patterns were almost symmetric about the two downstream spheres [5]. Saha et al. stated that, though the overall flow-past the square cylinder resembled that of the circular cylinder, there were major differences in terms of the separation mechanism and the related integral parameters such as Strouhal number, lift and drag-coefficient. Vortex-shedding frequencies and surface pressures of the square, circular and rectangular cylinders in a wind tunnel were investigated experimentally using hot-film measurement techniques. They determined the dominant peak location of the vortex shedding frequency and obtained Strouhal numbers in the range of various Reynolds numbers for the circular cylinder, square cylinder and oriented square cylinder [6].

In the last decade, computational resources have increased sufficiently such that high resolution solutions for practical engineering have become feasible. Numerical modeling for flow motion around bodies is based on methods of Computational Fluid Dynamics (CFD). There have been a lot of turbulence model to analysis flow field numerically. Beginning with low Re cases, researchers found excellent agreement with experimental values for most parameters monitored using a laminar Unsteady Reynolds-Averaged Navier-Stokes (URANS) method. Moving into the subcritical regime, research conducted by Rahman et al. compares a number of two-equation turbulence models at Reynolds of 1000 and 3900, and results show a clear improvement in agreement using the shear stress transport model (SST) over the $\mathrm{k}-\varepsilon$ and realizable $\mathrm{k}-\varepsilon$ models [7]. Benim et al. explored the topic of near wall meshing further by using the commercial code FLUENT to compute flow around a cylinder at $\mathrm{Re}=10^{4}$ using wall models and the standard $\mathrm{k}$ $\varepsilon$ turbulence model. They found acceptable correlation in the supercritical regime but this rapidly loses accuracy in the critical transition region, under-predicting values quantitatively for both $\mathrm{k}-\varepsilon$ and, to a lesser extent, SST models [8]. Stringer et al. developed and tested flow around circular cylinders at $\mathrm{Re}=40$ to $10^{6}$ with using different mesh methodology and appropriate turbulence modelling in Open-FOAM and ANSYS-CFX to find lift and drag forces, Strouhal frequency and boundary layer correlation. The practical solve times a 2D URANS Approach is taken. As a result of their previous study, have found successful application of this method for some of the Reynolds numbers, but a clear methodology for the entire flow field has not formerly been suggested and evaluated. At low Reynolds number, $\operatorname{Re}<10^{3}$, the method developed in the research is highly well-aimed with both solvers achieving correlation with experiment. However, at subcritical Reynolds numbers, $\mathrm{Re}=10^{5}$, the findings are less conclusive. Use of two solvers has uncovered basic differences despite closely matched definitions for this Reynolds numbers. They concluded that individual benchmarking of software is an essential step for any simulation, a requirement heightened in this case with increasing boundary layer and wake turbulence [9]. Rodi compared the LES and RANS calculations of the flow around square cylinder at $\mathrm{Re}=22000$ and for cube $\mathrm{Re}=40000$. He concluded that the various calculation results are compared with detailed experimental data and an assessment is given of the performance, the cost and the potential of the various methods. It is stated that LES is clearly more suited and has great potential for calculating these complex flows [10]. Dehkordi et al. investigated that the 2-D unsteady viscous flow around two circular cylinders in a tandem arrangement is numerically simulated in order to study the characteristics of the flow in both laminar and turbulent flow conditions. The applied computational method is firstly approved through simulation of laminar and turbulent flows around a fixed circular cylinder. The flow visualization parameters, the Strouhal numbers, and drag and lift coefficients are thoroughly presented and compared for different cases in order to reveal the effect of the Reynolds number and gap spacing on the behavior of the flow. The obtained results have shown two completely distinct flow characteristics in laminar and turbulent regimes [11].

The drag and lift coefficient of a cylinder is mainly affected by liquid viscosity, object size, velocity and a corresponding Reynolds number. Many methods have been used to determine these coefficients of a cylinder, e.g. load cell and numerical simulation. Recently, lots of researchers using numerical simulation, because the difficulties of experimental setup for force measurement systems, CFD can be applied to determine the load effects which acting on bluff bodies. Coupled with computer aided flow visualization, which provides visual animation, the numerical simulation may serve as a useful tool to analyze the evolution of flow field around structures and the attendant load effects. There are several studies on the literature about this subject. Juncu used the Navier-Stokes equations to analyse the hydrodynamic characteristics of two cylinders in tandem arrangements, and proposed that there was attempt between the two different cylinders in the same condition and the differences amplified with the increasing Re [12]. Itoh et al. investigated the effects of upwind scheme on the aerodynamic forces were investigated by comparison between LES with and without the use of an upwind scheme. The accuracy of LES without using an upwind scheme was clarified. The proposed method improves the standard deviation of pressure distributions around a circular cylinder at $\mathrm{Re}=10000$ [13].

Zou et al. analysed the turbulent flow around the four cylinder arrays with spacing ratios $\mathrm{L} / \mathrm{D}=1.5$ and 3.5 at a subcritical Reynolds number $10^{4}$ have been investigated using LES method and experimental techniques (LDA and DPIV). The vortex formation length for the upstream and downstream cylinders, pressure fluctuating and force 
coefficients are affected by the spacing ratio. 3-D numerical simulation using the LES method well replicates with the experimental features. It is a reliable method to reveal the full field quantitative information for any instance which may be extremely difficult to obtain by experimental investigation [14].

More recently, Tamura time transient large eddy simulation (LES) has become a powerful CFD tool for turbulent flow analysis as it resolves the large-scale unsteady motions and accounts for smaller eddies using a sub-grid-scale model [15]. Rahim et al. determined the free-surface effects on the wake structures downstream of a spherical body different submerging elevations with using LES methods at $\mathrm{Re}=5000$. They concluded this study with the results of a previous study done by Ozgoren . It is found that the numerical simulations were showed well agreement with the experimental results [1617].

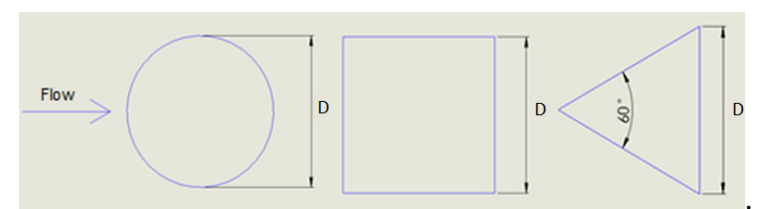

Figure 1. Different cross sectional cylinders.

The main aim of the present study is to investigate experimentally and numerically the flow field in the wake region of different bluff bodies such as circular, square and triangle cross section cylinders placed at horizontal position under the uniform flow condition (Figure 1). For experimental study Particle Image Velocimetry (PIV), for numerical simulation ANSYS-Fluent software are used. Also LES turbulence model was used to simulate flow behaviours and to obtain the drag coefficient numerically.

\section{Material Methods}

\subsection{Experimental Setup}

The experiments were carried out in a low-speed and large-scale open-loop water channel with a rectangular working section of $6000 \times 770 \times 600 \mathrm{~mm}$ in the facilities of Advanced Technology Research and Application Center of Selcuk University in Turkey. The walls of the test-section were made of $15 \mathrm{~mm}$ thick transparent acrylic and glass plates to facilitate laser transmission and flow visualization. An overview of the experimental system is shown in Figure 2. Before reaching the test chamber, the water was pumped into a settling chamber and passed through a honey-comb section. The Reynolds numbers of the external flow are calculated by, $\boldsymbol{R} \boldsymbol{e}=\boldsymbol{U}_{\infty} \boldsymbol{D} / \boldsymbol{v}$; based on the different cross sectional diameter of cylinders. Here, $v$ is the kinematic viscosity and $\boldsymbol{D}$ is characteristic length of cylinders. $\boldsymbol{U}_{\infty}$ is the free-stream velocity in the range of $100-230 \mathrm{~mm} / \mathrm{s}$. All cylinders were made of Plexiglas with the $5 \mathrm{~mm}$ thickness so that the laser light easily passes through the cylinders. The cylinders surfaces were highly polished to avoid the effects of surface roughness. The cylinders had a hollow section in the symmetry plane and the section was filled with water to minimize the laser light deflection. The laser sheet was located vertically from the channel bottom side as seen in Fig. 2 while the water height was $h_{W}=450 \mathrm{~mm}$ in all cases and cylinders located at $225 \mathrm{~mm}$ position above channel bottom surface. Nd:YAG laser was used to generate a laser sheet that was perpendicular to the axis of the cylinder was passed through them with 1000 pulse per second, and the illuminating laser sheet thickness in the flow field was nearly $1 \mathrm{~mm}$. A CMOS camera with a resolution of $1632 \times 1200$ pixels was used to record the images. As illustrated in Fig. 2, the laser was attached in a fixed position underneath the water channel while the camera was the right angle to the laser sheet.

The densities of the particles and water were close enough, and the suspended seeding particles with a diameter of $10 \mu \mathrm{m}$ in the flow were silver coated hollow glass spheres. Furthermore, the high-image-density criterion was contented by ensuring that a minimum of approximately 20-30 particles was contained within the interrogation area. Dynamic Studio software employing the adaptive correlation algorithm including proper filters was used for computing the raw displacement vector field from the particle image data. An interrogation window of $32 \times 32$ pixels in the image was selected and converted to approximately $2.0 \times 2.0 \mathrm{~mm}^{2}$ grid size consisting of 7400 $(100 \times 74)$ velocity vectors. During the interrogation process, an overlap of $50 \%$ was employed in order to satisfy the Nyquist criterion. Patterns of instantaneous particle images with a 3700 images consisting of a continuous series were taken at the rate of $500 \mathrm{~Hz}$ to calculate the time-averaged patterns of the flow structure.
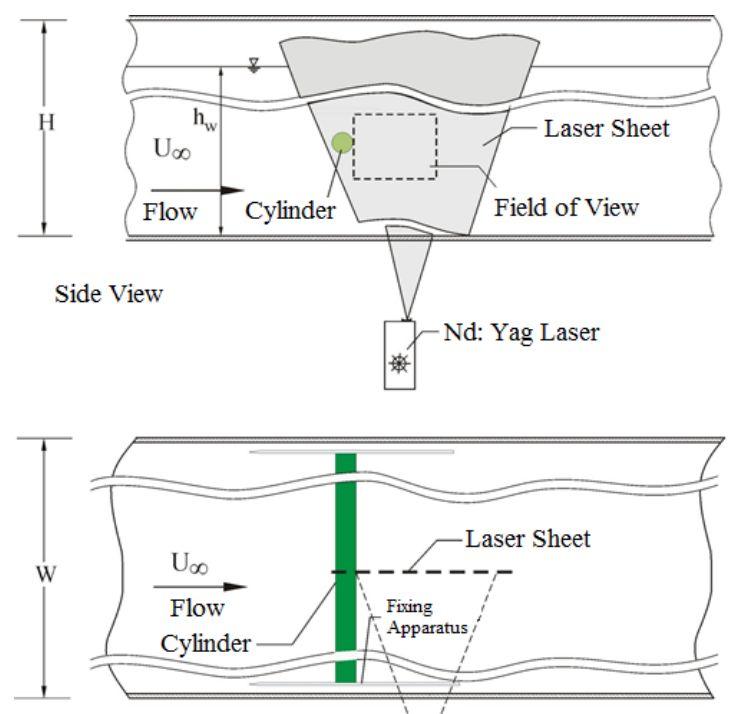

Plane View

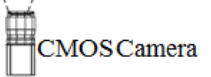

Figure 2. Schematic view of the experimental setup of PIV system, laser illumination for a cylinder located in a uniform flow condition.

\subsection{Numerical Simulation}

In the present study, 3-D water flow above the different cross sectional cylinders at Reynolds number 5000 and 10000 was simulated with ANSYS Fluent 14.5. The 
dimensions of flow field are same with the experimental flow volume, $-4 \mathrm{D}<\mathrm{x}<16 \mathrm{D}$ in the stream-wise direction, $4.5 \mathrm{D}<\mathrm{y}<4.5 \mathrm{D}$ in the cross stream direction and $6 \mathrm{D}<\mathrm{z}<6 \mathrm{D}$ in the perpendicular direction to the $\mathrm{x}-\mathrm{y}$ plane. All the cylinders were located at the origin of coordinate system. A cut-cell grid system was used for all cylinders model (Figure 3). In the generation of grid system, coarsen mesh was applied around and the wake region of the bluff body since highly complexity of the flow. On account of grid independency, different number of grid was applied up to 14 million cells each geometry. With the rising of cell numbers, however, the accuracy of the flow data was not changed and solution process was taken more time. Between the 8-10 million, analyses were completed.

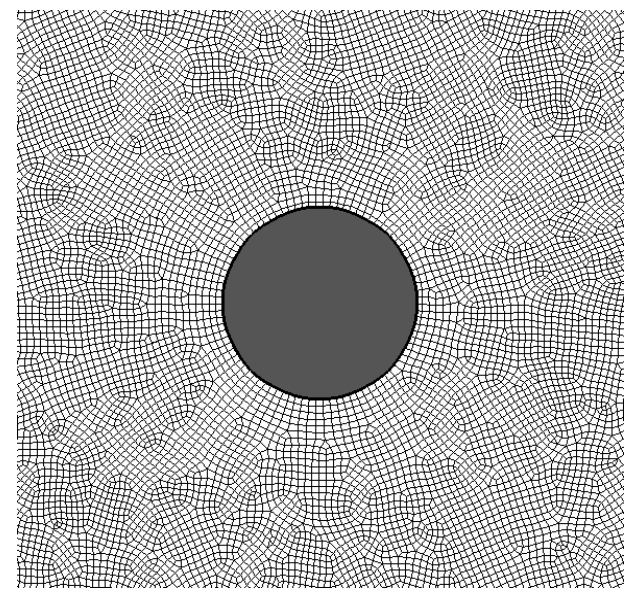

Figure 3. Cut-cell grid system around circular cylinders

For the numerical investigations, LES technique was applied, which resolves most of the turbulent length scales. Just the small-scale structures of turbulence were modelled. LES is the process in which instead of modeling all scales such as RANS, separation of large eddy from smaller ones is under emphasis using a filtering process. Based on this approach, the smaller scales of motion are universal and isotropic and so they can be modeled according to Kolmogorov principles [19].

The LES code solves the filtered continuity equation and the filtered incompressible Navier-Stokes equations as follows

$$
\frac{\partial \overline{u_{i}}}{\partial x_{i}}=0
$$

$$
\frac{\partial \overline{u_{i}}}{\partial t}+\frac{\partial \overline{u_{i}} \overline{u_{j}}}{\partial x_{j}}=\frac{1}{p} \frac{\partial \bar{p}}{\partial x_{i}}-\frac{\partial\left(\overline{u_{i}} \overline{u_{j}}-\overline{u_{i}} \cdot \overline{u_{j}}\right)}{\partial x_{j}}+v \frac{\partial^{2} \overline{u_{i}}}{\partial x_{i} \partial x_{j}}
$$

where $\boldsymbol{t}, \boldsymbol{x}_{\boldsymbol{i}}, \boldsymbol{u}_{\boldsymbol{i}}$ and $\boldsymbol{v}$ are the time, the Cartesian coordinates, velocity components and the kinematic viscosity, respectively.

For the purpose of resolve the Navier-Stokes equations of motion (Eq. (1)) and continuity equation (Eq. (2)), the finite volume method is used in which the general scalar transport equation for transient and 3-D flow condition is presented by

$$
\begin{gathered}
\int_{C V}\left(\int_{t}^{t+\Delta t} \frac{\partial}{\partial t}(\rho \varnothing) d t\right)+\int_{t}^{t+\Delta t}\left(\int_{A} n(\rho u \emptyset) d A\right) d t \\
=\int_{t}^{t+\Delta t}\left(\int_{A} n(\Gamma g r a d \Phi) d A\right) d t \\
+\int_{t}^{t+\Delta t}\left(\int_{C V} S_{\phi} d V\right) d t
\end{gathered}
$$

where $\boldsymbol{\Gamma}, \boldsymbol{\Phi}$ and $\boldsymbol{S}_{\boldsymbol{\Phi}}$ are the diffusion coefficient, general property and growth rate of $\boldsymbol{\Phi}$ owing to sources, respectively [20]. Order of accuracy of the flow model in space and time also affects the solution accuracy. A uniform inflow condition with constant flow speed $\boldsymbol{U}_{\infty}$ was used for inlet boundary condition. Also lateral, bottom and the top surfaces of the flow volumes boundary conditions were the same conditions of the inlet with the constant velocity through the stream wise direction. The outlet was sampled as an outflow, allowing for vortices to pass the boundary without being reflected. During the solution, time step $\Delta \mathrm{t}=0,0016 \mathrm{~s}$ was applied constant and the same with experimental data. Each time step occurs of 10 sub-iterations and simulations are executed up to 3700 time steps. Furthermore, the convergence of the numerical 3-D velocity components was set up at each time step by examining the residuals of all equations to be solved by setting their variations less than $10^{-7}$.

Finally, for processing of numerical simulation result ANSYS CFD-Post was used. Instantaneous and time averaged velocity contours, vorticity, streamline topology and kinetic energy values were obtained graphically. In addition, flow characteristics like drag coefficient and pressure coefficient of the geometries were also calculated.

Instantaneous values of drag coefficient $\mathrm{C}_{\mathrm{D}}$ using Eqs. (4), where $F_{D}$ is the corresponding unit forces act on cylinders surface [21].

$$
C_{D}=\frac{F_{D}}{0.5 \rho U^{2} A}
$$

\section{Results}

In this section, some of the most relevant data computed are submitted and discussed with respect to the experimental data as well as comparatively to eachothers. The full range of conditions expected in the case of a cylinder in uniform flow for experimental and numerical calculations have been performed at $\mathrm{Re}=5000$ and $\mathrm{Re}=10000$. Time-averaged flow patterns are given in Figure 4 each cross sectional cylinders. All figure dimensions are normalized with the appearance characteristics length as $\mathrm{x} / \mathrm{D}$. Layers of positive and negative values are displayed with blue and red contours, respectively. From time-averaged experimental and numerical analysis streamlines topology, it can be clearly seen that the wake region length at $\mathrm{Re}=5000$ is longer than $\mathrm{Re}=10000$. For square cross sectional cylinder, two symmetrical foci were formed at the trailing edge with 
the length of nearly $0.7<\mathrm{x} / \mathrm{D}<0.8$ and $0.5<\mathrm{x} / \mathrm{D}<0.6$ at $\mathrm{Re}=5000$ and 10000 , respectively. In addition, there were also two small foci occurred on the lower and upper surface of square. These foci were seen clearly from numerical analysis results, and foci at low Reynolds number were thick and short, for high Reynolds number vice versa. However, these foci from experimental results were not able to be viewed because of their sharp edges cause reflections near the surfaces. The second geometry, circular cylinder, two symmetrical foci were detected in the wake region both experimental and numerical results for each Reynolds number.

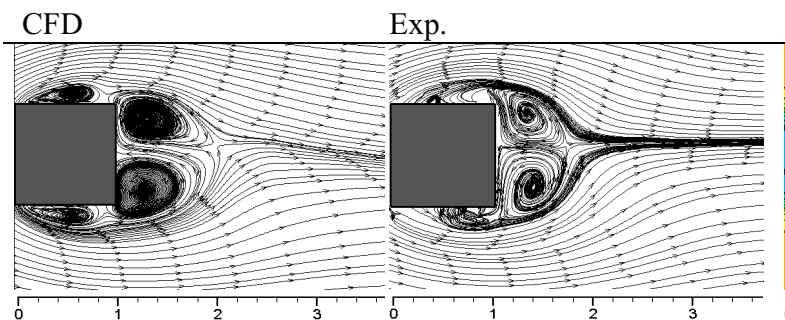

CFD Exp.
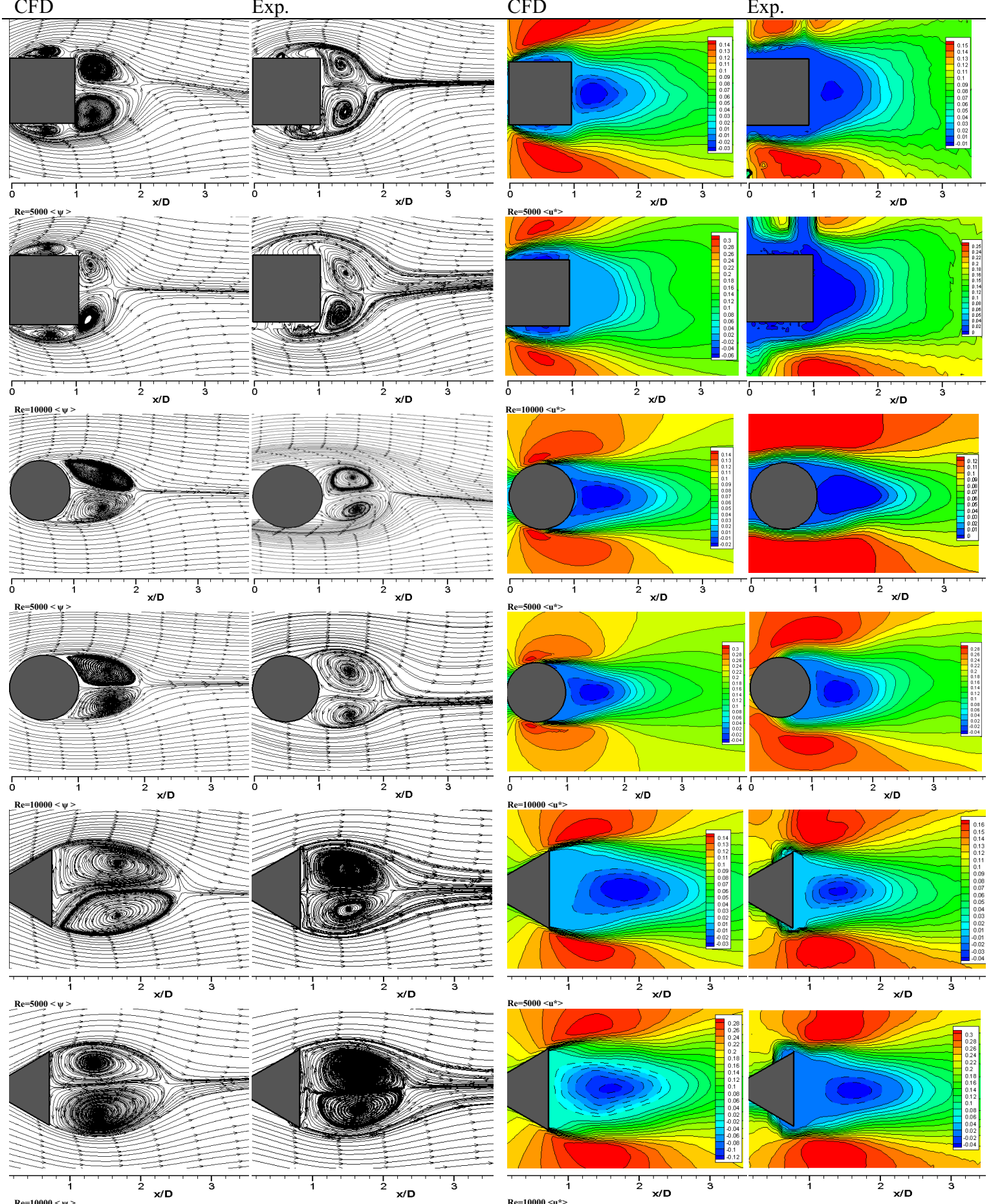

Figure 4. Flow patterns of the normalized time-averaged streamline $(\langle\psi\rangle)$ and stream wise velocity $\left.\left(<\mathrm{u}^{*}\right\rangle\right)$ components of experimental (right column) and numerical analysis (left column) results at $\mathrm{Re}=5000$ and 10000 around different cross-sectional cylinders.

At $\mathrm{Re}=5000$, length of foci from the base of the circular cylinder was in the range of $0.9<\mathrm{x} / \mathrm{D}<1.0$ while for $\mathrm{Re}=10000$ foci were about $0.8<\mathrm{x} / \mathrm{D}<0.9$. The separation point from the surface of the circular cylinder was occurred before $90^{\circ}$ from the front stagnation point. For the equilateral triangle cylinder, the spike point of triangle was located contrary to the stream wise direction. From experimental results, for each Reynolds 
number the length of wake region had similar results which were about $1.8<\mathrm{x} / \mathrm{D}<1.9$. While the numerical results show that the length of wake region was longer at low Reynolds number with $2.0<\mathrm{x} / \mathrm{D}<2.1$ and at $\mathrm{Re}=10000$ it was $1.9<\mathrm{x} / \mathrm{D}<2$.

In this study, both experimental and numerical analysis results show that the wake length of the triangle cylinder was the longest and the square cylinder was the shortest. As a result of experimental and LES analyses, the time-averaged stream wise velocity components were very close appearance. When the contour scalar of both results were analysed, each matching was clearly observed similar velocity value. In wake region, the stream wise velocity had received negative values for both results. From these images, it is also possible to define the location of a stagnation point around the wake symmetry plane.
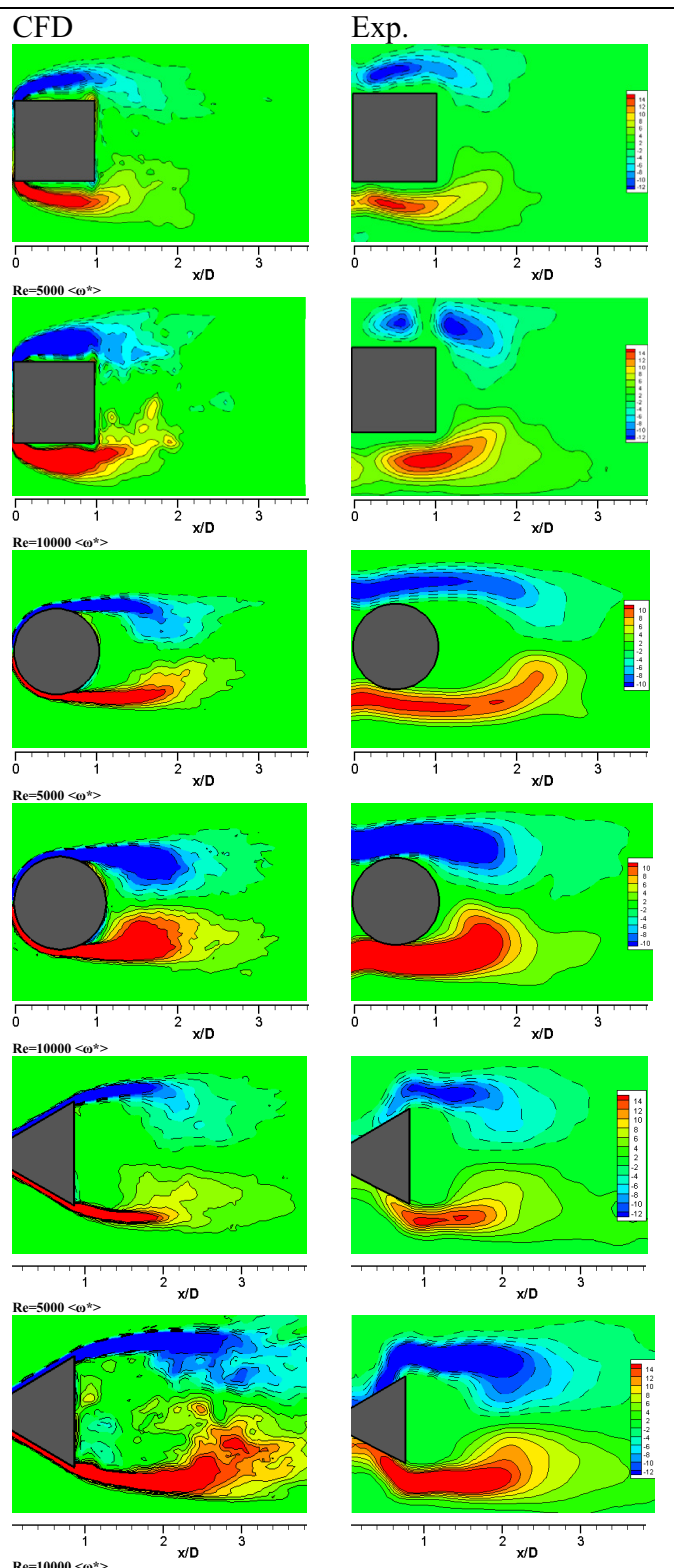

Figure 5. Time-averaged vorticity components $\left(<\omega^{*}>\right)$ of numerical analysis (left column) and experimental (right column) results at $\mathrm{Re}=5000$ and 10000 around different crosssectional cylinders.
In Figure 5, time-average vorticity contour is seen, the numerical analysis results are shown on the left column, and the other is the experimental. Two symmetrical vortices were occurred around the bluff bodies, one was counter clockwise defined as positive, and the other was in the opposite direction. Generally, at high Reynolds numbers, length of vorticity was bigger for all cylinders, higher values of vorticity contours covers a wider area and the flow was more chaotic. For square cylinder the vorticity was started from leading edge, and unlike the other geometry near the upper and lower surfaces of the body vorticity contours were getting high values. Positive values are more dominant compared to negative values with the max. $14 \mathrm{~s}^{-1}$ and min. $-12 \mathrm{~s}^{-1}$. For circular cylinder, vorticity contours were along the shear layer. At $\mathrm{Re}=5000$ time averaged vorticity contours form trailing edge were comprised further away from the cylinder base at $\mathrm{Re}=10000$. The other geometry, triangle cylinder, time-averaged vorticity contours were began to be seen at the endpoints of trailing edge. At $\mathrm{Re}=10000$ fluctuations were seen higher on the contrary to the circular cylinder time averaged vorticity contours form trailing edge were comprised further away from the cylinder base at $\mathrm{Re}=5000$. These vorticity fluctuations induce unsteady forces on the bluff body. Another aim of presented study was determine the Drag Coefficient $\left(\mathrm{C}_{\mathrm{d}}\right)$ of different type cross sectional cylinders. As a result of experimental and LES analyses, in Figure 4 and 5, the time-averaged stream wise velocity components, the streamline topology and the vortices were in close agreement. After comparing numerical analysis and experimental data with well agreement, the drag coefficient was found using LES turbulence model. From Figure 6, the time-averaged and instantaneous drag coefficient for the circular cylinder at $\mathrm{Re}=10000$ is given. After providing uniform flow conditions, considering experimental time-step, which is $0,0016 \mathrm{~s}$ with $3700 \mathrm{step}$, the numerical analysis completed and the average was found to be $\mathrm{C}_{\mathrm{d}}=1,01$. This result was overlapped with Schlichtings' findings [22].

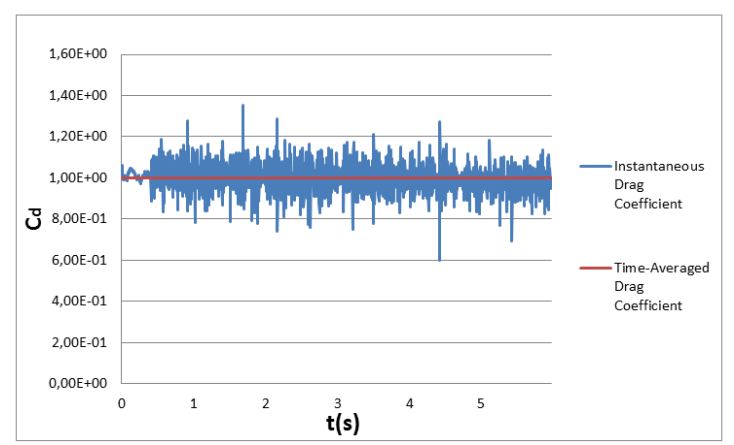

Figure 6. The time history of drag coefficient for circular cylinders at $\mathrm{Re}=10000$

This method was also applied for all bluff bodies at two Reynolds numbers, and results were presented in Table 1. It shows that there were no significant differences within the drag coefficient at both Reynolds numbers for square and circular cylinders. However, 
this situation is different for triangle cylinder. Drag coefficient at $\mathrm{Re}=5000$ was greater than $\mathrm{Re}=10000$. When compared all geometries, in both Reynolds numbers the square cylinder had the greatest value for each characteristic. The results of the square cylinder are also matching with the literature [23].

Table 1. Time-averaged Drag Coefficient of cylinders

\begin{tabular}{|c|c|c|}
\hline & $\mathrm{C}_{\mathrm{d}} / \mathrm{Re}=5000$ & $\mathrm{C}_{\mathrm{d}} / \mathrm{Re}=10000$ \\
\hline Square Cylinder & 1,77 & 1,78 \\
\hline Circular Cylinder & 1,08 & 1,01 \\
\hline Triangle Cylinder & 1,62 & 1,36 \\
\hline
\end{tabular}

\section{Conclusions}

In present study, the wake region of different bluff bodies such as circular, square and triangle cross section cylinders placed horizontally perpendicular position to the uniform flow were investigated experimentally and numerically. In addition, flow characteristics like drag coefficient of the geometries were also calculated. For experimental study Particle Image Velocimetry (PIV), for numerical simulation ANSYS-Fluent software were used. LES turbulence model utilized for the numerical simulation and the drag coefficient was obtained from this simulation. The full range of conditions expected in the case of a cylinder in uniform flow for experimental and numerical calculations had been performed at $\mathrm{Re}=5000$ and 10000 .

From time-averaged experimental and numerical analysis results, the wake length at $\mathrm{Re}=5000$ is longer than $\mathrm{Re}=10000$ for all cylinders. Both experimental and numerical analysis results show that the wake length of the triangle cylinder was the longest and the square cylinder was the shortest. As a result of experimental and LES analyses, the time-averaged stream wise velocity components were in close agreement. In wake region, the streamwise velocity had received negative values for both results. Results show that, symmetrical two vortices were occurred around the bluff bodies one was positively and the other was in the opposite direction. Generally, at high Reynolds numbers, length of vorticity was bigger for all cylinders, higher values of vorticity contours covers a wider area and the flow was more chaotic. LES results are validated against experimental measurements, and reasonable agreements are obtained as shown in Figures 4 and 5 by the timeaveraged stream wise velocity components, streamline topology and vortices. At $\mathrm{Re}=10000$, the drag coefficient of the circular cylinder from the specific literature was yielded similar results and this method might be used for further analyses. At both Reynolds numbers, there were no significant differences within the drag coefficient for square and circular cylinders. For the triangle cylinder, drag coefficient was greater at $\mathrm{Re}=5000$. When all geometries were compared, either $\mathrm{Re}=5000$ or $\mathrm{Re}=10000$ the square cylinder had been taken the greatest values and circular cylinder was the smallest. The obtained and validated results can be useful for designers.
Acknowledgments: Institutional supports were made by Scientific Research Project Office of Selcuk University (project numbers of 11401058 and 11401059) and Government Planning Organization (DPT) via the $2009 \mathrm{~K} 12180$ coded project of Advanced Technology Research and Application Center. Selcuk University Academic Staff Training Program provided the international conference attendance support for Experimental Fluid Mechanics Symposium 2014.

\section{References}

1. M. Ozgoren, Flow Measurement and Instrumentation, 17 (2006)

2. Okajima A., Journal Fluid Mechanic, 123 (1982)

3. M.S. Kim, D. Geropp, J. of Wind Engineering and Industrial Aerodynamics, 74-76 (1998)

4. K. Lam, J.Y. Li, K.T. Chan, R.M.C., Journal of Fluid Structures, 17 (2013)

5. M. Ozgoren, International Journal of Multiphase Flow, 53 (2013)

6. Saha A.K., MuralidhaK., Biswas G., Experimental Fluid Mechanics, 29 (2000)

7. Rahman, M.M., Karim, M.M., Alim, M.A., J. Nav. Archit. Mar. Eng., 4 (2008)

8. Benim, A.C., Cagan, M., Nahavandi, A., Pasqualotto, E., Proceedings of the 5th Iasme/Wseas International Conference on Fluid Mechanics and Aerodynamics, (2007)

9. R.M. Stringer, J.Zang , A.J.Hillis, Ocean Engineering, 87 (2014)

10. W. Rodi, Journal of Wind Engineering and Industrial Aerodynamics, 69-71 (1997)

11. Dehkordi B. G., Moghaddam H. S., Jafari H. H., Journal of Hydrodynamics, 23(1) (2011)

12. Juncu G., Internaional Journal of Heat and Mass Transfer, 50 (2007)

13. Itoh Y. and Tamura T., J. of Wind Engineering and Industrial Aerodynamics, 96 (2008)

14. Zou L., Lin Y., Lam K., Journal of Hydrodynamics, 20(4) (2008)

15. Tamura T., Journal of Wind Engineering and Industrial Aerodynamics, 96 (2008)

16. R. Hassanzadeh, B. Sahin, M. Ozgoren, Ocean Engineering, 54 (2012)

17. Ozgoren M., Pinar E., Sahin B., Akilli H., Journal of Fluid Mechanics, 572 (2011)

18. R. Hassanzadeh, B. Sahin, M. Ozgoren, Int. J. of Computational Fluid Dynamics, 25(10), (2011)

19. M. Ozgoren and S.Dogan, Experimental Fluid Mechanics, pp.363-376 (EFM2011)

20. Rodney C.S., Alan R.K., Randall M., Computer Methods in Applied Mechanics and Engineering, 199 (2010)

21. Vakil A., Green S.I., Computers and Fluids, 38 (2009)

22. Schlichtings H., Fluid Mechanics, Boundary Layer Theory, 7 (1979)

23. Tetsuro T., Tetsuya M., Journal of Wind Engineering and Industrial Aerodynamics, 83 (1999) 